\title{
LIMITES DE DEFORMAÇÃO (M) PARA VALIDADE DA MECÂNICA DA FRATURA ELASTO-PLÁSTICA MONOPARAMÉTRICA (INTEGRAL $J$ ) EM CORPOS DE PROVAS SE(B), C(T) E SE(T)*
}

Gustavo Henrique Bolognesi Donato ${ }^{1}$ Felipe Cavalheiro Moreira ${ }^{2}$

\section{Resumo}

Este trabalho apresenta o desenvolvimento de critérios objetivos para a garantia de validade da mecânica da fratura elasto-plástica monoparamétrica, utilizando o parâmetro integral $J$ como descritor dos campos de tensões, deformações e deslocamentos à frente de um defeito do tipo trinca. O mais importante na vertente monoparamétrica da mecânica da fratura é garantir que os campos de tensões no corpo de prova laboratorial sejam comparáveis com os existentes em uma estrutura real para cujo projeto as propriedades retiradas do corpo de prova serão utilizadas (conceito este denominado similitude). Para estabelecer critérios objetivos para a existência de similitude, esse trabalho comparou os campos de tensões obtidos de estruturas de referência sob alta triaxialidade de tensões ( $M B L$ ) com aqueles obtidos de corpos de provas de mecânica da fratura em escala de laboratório. A matriz de análise permitiu a determinação dos limites de deformação $M$ para geometrias $\mathrm{C}(\mathrm{T}), \mathrm{SE}(\mathrm{B})$ e $\mathrm{SE}(\mathrm{T})$ de diversas configurações e para uma faixa de propriedades de material característica de aços estruturais aplicáveis a vasos de pressão e dutos. Os resultados demonstram a baixa restrição à plasticidade de corpos de prova $S E(B)$ e $S E(T)$ com trincas rasas e os efeitos de profundidade de trinca e espessura nos resultados de $M$. Ainda, alguns comportamentos inesperados foram evidenciados, como no caso em que (para condições particulares de profundidade de trincas) corpos de prova mais finos apresentaram maior restrição a plasticidade do que amostras mais espessas. Sendo assim, o trabalho oferece uma base objetiva para que a similitude seja garantida nas avaliações de integridade estrutural baseadas na mecânica da fratura elasto-plástica suportada pela integral $J$, seja com seus valores críticos $(J c)$ ou curvas $J-R$.

Palavras-chave: Limite de deformação; Modelo da camada limite; Integral $\mathrm{J}$; Similitude.

\section{DEFORMATION LIMITS $(M)$ FOR THE ONE-PARAMETER ELASTIC-PLASTIC FRACTURE MECHANICS ( $J$ INTEGRAL) VALIDITY ON SE(B), C(T) AND CLAMPED SE(T) SPECIMENS}

\begin{abstract}
This work presents the development of objective criteria to ensure the one-parameter elastic-plastic fracture mechanics validity using the $J$ integral to describe the stress/strain/displacement-fields ahead of crack-like defects. The most important aspect of the one-parameter fracture mechanics framework is to ensure that the stress-fields in a reduced laboratory specimen are comparable to those found in real structures (this is the so-called similitude concept). To establish objective criteria for the similitude existence in the analysis, this work compared the stress-fields obtained from high constraint reference models $(M B L)$ with those obtained from laboratory scale fracture mechanics specimens. The analysis matrix allowed the determination of the deformation limits $M$ for $\mathrm{C}(\mathrm{T})$, $\mathrm{SE}(\mathrm{B})$ and clamped $\mathrm{SE}(\mathrm{T})$ geometries considering a wide range of geometrical features and material properties characteristic of structural steels applicable to pressure vessels and pipelines. The results confirmed the low constraint response for short-cracked $S E(B)$ and $S E(T)$ specimens and clarified the effects of crack depth and thickness on $M$ values. In addition, some unexpected behaviors were observed, as the case in which (for some particular crack depths) thin specimens are more constrained than thick ones. Thus, this work provides insights for the development of an objective basis to guarantee similitude in structural integrity assessments based on elastic-plastic fracture mechanics supported by the $J$ integral either with its critical values $(J c)$ or $J-R$ curves.
\end{abstract}

Keywords: Deformation limit, MBL model; $J$ integral; Similitude.

1 Engenheiro Mecânico, Doutor, Docente do Departamento de Engenharia Mecânica do Centro Universitário da FEl, São Bernardo do Campo, SP, Brasil, gdonato@fei.edu.br

2 Engenheiro Mecânico, Mestre, Centro Universitário da FEl, São Bernardo do Campo, SP, Brasil. 


\section{INTRODUÇÃO}

Se consideradas as soluções estruturais adotadas nos dias atuais, ficam evidentes geometrias com reduzidas espessuras e otimização geométrica. Como consequência, maiores níveis de tensões, deformações e deslocamentos podem atuar em componentes mecânicos, gerando assim maior severidade de operação e riscos no caso da existência de defeitos. Como resposta a este cenário de elevados carregamentos, os materiais de uso corrente apresentam, em sua maioria, elevadas resistência mecânica e tenacidade combinadas a grande capacidade de deformação plástica precedente à falha. Esse aumento de plasticidade exige a aplicação da Mecânica da Fratura Elasto-Plástica (MFEP) e deriva daí a importância de investigar os limites de validade de seus parâmetros (especialmente $J$ ) para previsões de falha. De forma central, mais importante do que descrever o campo de tensões em um corpo de prova laboratorial de dimensões reduzidas, é garantir que o campo de tensões presente nesse corpo de prova seja o mesmo que o existente em uma estrutura real para cuja avaliação as propriedades retiradas do corpo de prova serão utilizadas (conceito de similitude - figura 1). Tal ocorrência valida o emprego da mecânica da fratura monoparamétrica, a qual admite que um único parâmetro possa descrever o campo de tensões na frente de uma trinca em uma estrutura ou corpo de prova [1].



Figura 1. Conceito de similitude e mecânica da fratura elasto-plástica monoparamétrica. (a) Corpo de prova $\mathrm{C}(\mathrm{T})$; (b) estrutura solicitada contendo uma trinca e em condições de escoamento de pequena monta (SSY); (c) Zona de dominância $J$ na região da ponta da trinca e respectiva zona plástica. [1]

O foco de estudo do presente trabalho, portanto, está na utilização da MFEP monoparamétrica, governada pelo parâmetro denominado integral $J$ [2] para avaliação de falha de projetos mecânicos. Landes e Begley [3] foram os primeiros pesquisadores a propor um critério de falha baseado em parâmetros elasto-plásticos. O método consistia na avaliação de integridade estrutural baseada em tensões e governada exclusivamente pelo parâmetro integral $J$, isto porque o mesmo era univocamente relacionável com os campos de tensões apresentados por uma estrutura trincada carregada monotonicamente $[4,5]$. Entretanto, para que o valor de $J$ seja válido, ou seja, para que ele descreva os campos de tensões à frente da trinca e assim possa ser caracterizado como propriedade do material, é necessário que o mesmo esteja dentro de sua validade e para isso é necessário que algumas premissas sejam 
obedecidas, dentre elas carregamento monotônico e condições de plasticidade restrita na região da trinca. A perda de restrição à plasticidade é a causa mais usual da invalidação da integral $J$ pois torna as propriedades dependentes não somente do material sendo testado, mas também da geometria da amostra e carregamento.

Apresentado o cenário anterior, a motivação desse trabalho é centralizada na necessidade de se estabelecer critérios robustos e objetivos para a utilização e validade do parâmetro integral $J$ como descritor dos campos de tensões à frente de uma trinca qualquer, utilizando como base a comparação dos campos de tensões de estruturas de referência (modelos MBL sob estado de elevada restrição à plasticidade) e a de corpos de provas de mecânica da fratura simulados utilizando o Método dos Elementos Finitos (MEF). O objetivo final é estabelecer os limites de aplicabilidade da MFEP monoparamétrica com base em $J$, garantindo a existência de similitude e a decorrente transferabilidade de propriedades entre o laboratório e as aplicações reais.

\subsection{Dominância $J$ e o parâmetro $M$}

Paris, a partir de estudos de Landes e Begley [3] no ASTM STP 514 [7], foi o primeiro a sugerir uma tratativa para os limites de validade do parâmetro integral $J$ dentro do escopo da mecânica da fratura monoparamétrica. Posteriormente, diversas pesquisas no assunto $[8,9,10]$ aplicaram $J$ como critério de avaliação de falha em condições elasto-plásticas e demonstraram efeitos de plasticidade e outros na sua validade.

Por fim, Shih e German [11], sistematizaram as condições para a utilização da integral $J$ dentro de condições de dominância (descrição dos campos de tensões) na forma:

$$
B, b \geq M \frac{J}{\sigma_{0}},
$$

onde $B$ é a espessura do corpo de prova, $b$ é o ligamento remanescente, $\sigma_{0}$ é uma tensão de referência que pode ser a tensão limite de escoamento $\sigma_{L E}$ ou a média entre a tensão limite de escoamento e a tensão limite de resistência (denominada $\sigma_{\text {flow }}$ ).

Verificou-se após extensa revisão bibliográfica que a escassez de resultados para a tratativa dos limites de aplicabilidade da MFEP monoparamétrica, utilizando os limites de deformação $M$, é expressiva. Sabe-se também pela literatura que a sensibilidade do parâmetro a fatores como a geometria de corpos de prova, o comprimento relativo de trinca, o modo de carregamento e as propriedades de material é expressiva e por isso é de extrema importância que seja conhecida e estudada de maneira estruturada.

\section{MATERIAIS E MÉTODOS}

Como um passo na direção do estudo dos limites de deformação em corpos de prova padronizados para a aplicabilidade da mecânica da fratura monoparamétrica, optouse pela utilização de três geometrias amplamente conhecidas para a caracterização de tenacidade à fratura de materiais estruturais. As duas primeiras geometrias são conhecidas como Compact under Tension ou C(T) (figura 2a) e como Single Edge notched under Bending ou SE(B) (figura $2 b$ ) e são amplamente utilizadas pela norma ASTM E1820 [12]. A terceira geometria estudada, embora não seja padronizada pela norma anterior, pode ser encontrada na norma DNV-RP-F108 [13] e é conhecida como Single Edge notched under Tension ou SE(T) (figura 2c). Cada uma das geometrias apresenta comportamentos distintos em termos de carregamento, restrição plástica e, portanto, é de se esperar que os limites de validade da integral $J$ sejam diferentes para cada uma das geometrias consideradas. 

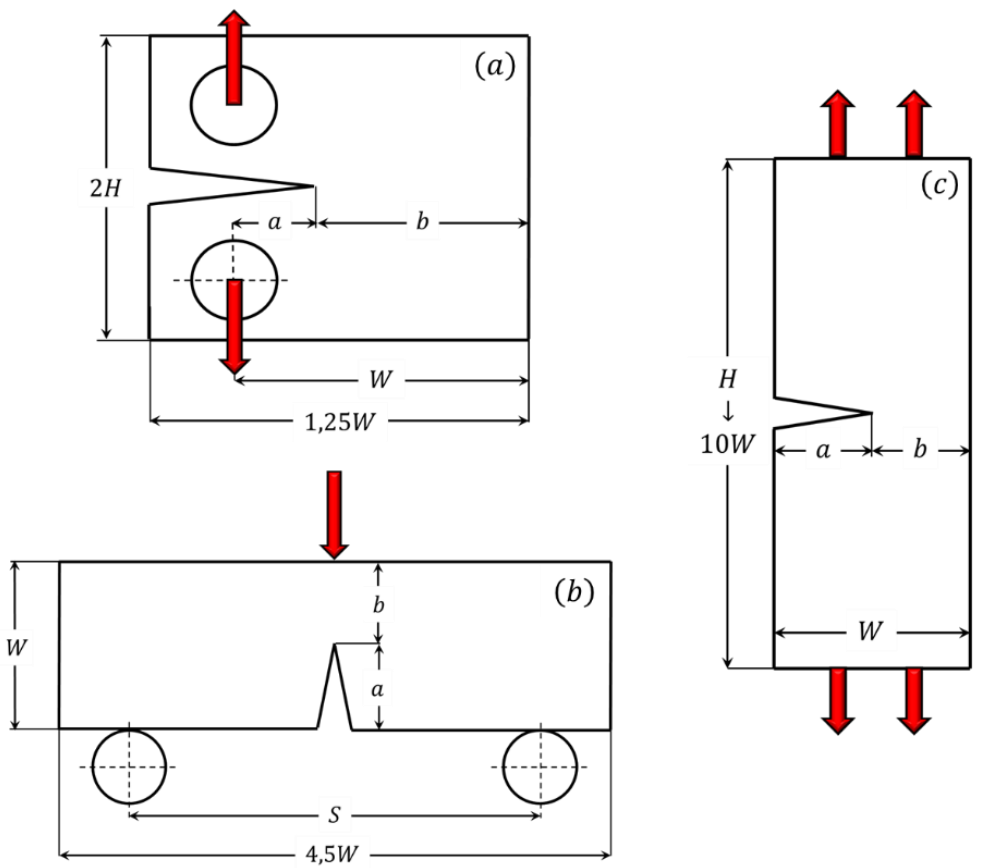

Figura 2. Principais corpos de prova considerados e suas respectivas dimensões planares.

\subsection{Modelos de elementos finitos desenvolvidos}

\subsubsection{Modelo elasto-plástico para corpos de prova}

As simetrias existentes permitem o desenvolvimento de modelos $1 / 2$ ou $1 / 4$ simétricos dos corpos de prova, desde que condições de contorno adequadas sejam impostas a todos os planos de simetria (o que significa deslocamentos nulos na direção normal aos referidos planos - vide figura 3 ).

O código de elementos finitos WARP3D [14] fornece a solução numérica para as análises elásticas e elasto-plásticas dos modelos desenvolvidos nesse trabalho incorporando teoria de plasticidade incremental $\left(J_{2}\right)$ tanto para teoria de pequenas (linear) como grandes deformações (não-linear). As propriedades dos materiais empregados são representativas de uma faixa bastante significativa de aços estruturais ferríticos utilizados em componentes estruturais, dutos e vasos de pressão. Assim, as três classes de materiais investigadas são apresentadas na tabela 1.

Tabela 1. Propriedades dos materiais empregados nas análises de elementos finitos para todos os corpos de prova investigados. Vale lembrar que $n$ é o inverso do expoente $(N)$ do material $(n=1 / N)$

\begin{tabular}{lccc}
\hline $\boldsymbol{n}$ & $\boldsymbol{E} / \boldsymbol{\sigma}_{\mathbf{0}}$ & $\boldsymbol{E}(\mathbf{M P a})$ & $\boldsymbol{v}$ \\
\cline { 1 - 2 } 5 & 800 & & \\
\cline { 1 - 2 } 20 & 500 & & \\
\cline { 1 - 2 } 20 & 300 & & 0,3 \\
\hline
\end{tabular}

Todos os modelos de elementos finitos foram construídos no programa comercial de pré-processamento MSC Patran [15]. Visando obter de forma acurada a variação singular dos campos de tensões na região à frente da trinca, utilizou-se uma malha altamente refinada em todos os modelos. Nas proximidades do defeito, a malha é constituída de anéis focais centrados na ponta da trinca (figura 3 ) descrita em todos os casos por um raio inicial $\rho=0,0025 \mathrm{~mm}$ 
(a)

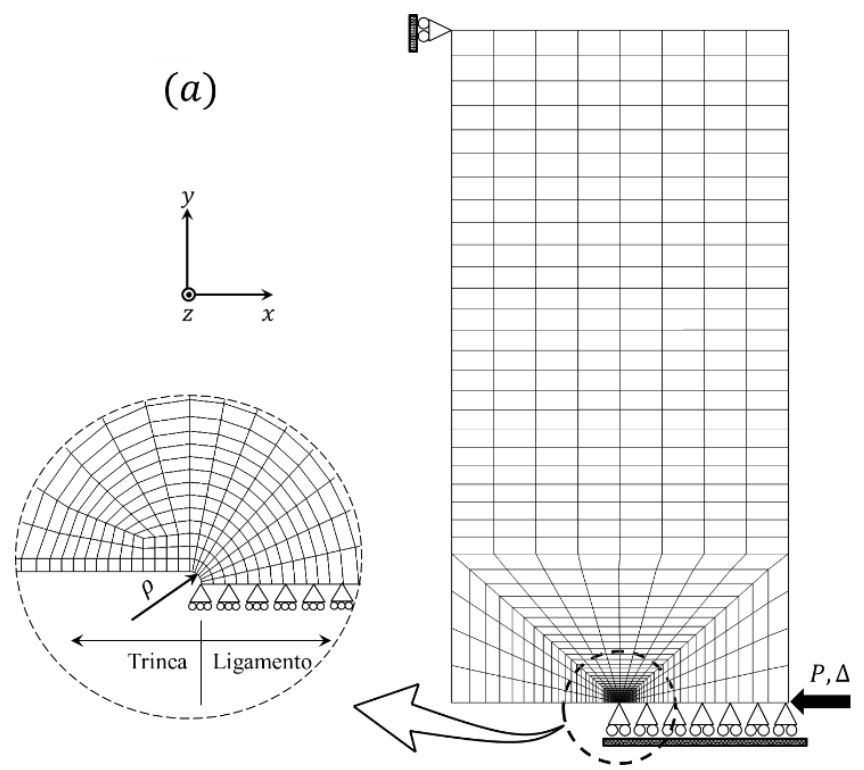

\section{降}

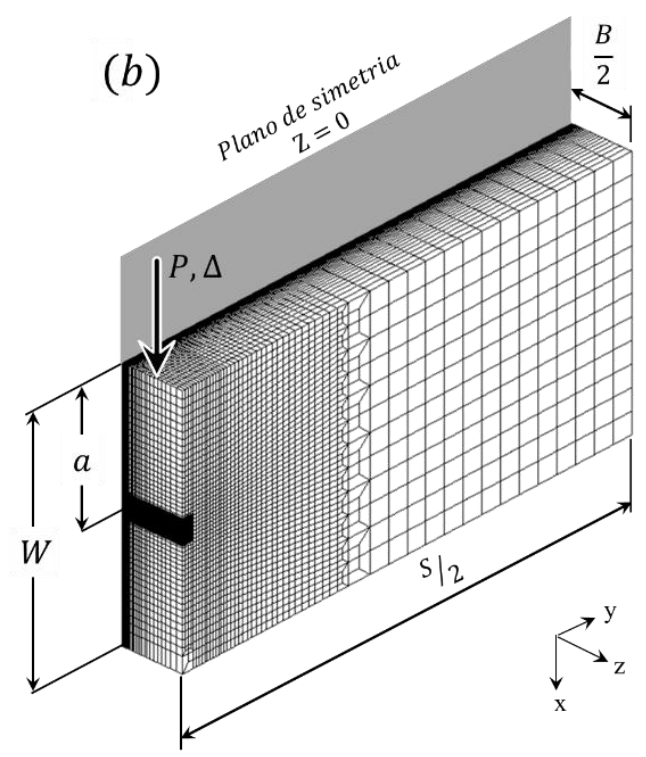

Figura 3. Padrão de malha e vinculação dos modelos MEF representativos de geometria $S E(B)$.

Primeiramente foram elaborados modelos sob Estado Plano de Deformações (EPD), com camada unitária de elementos 3D hexaédricos de 8 nós têm sendo os deslocamentos na direção $z$ restritos $(w=0)$ (figura 3a). Foram construídos também modelos de corpos de prova em contexto 3D pleno (figura 3b). No caso 3D, a metade da espessura $(B / 2)$ foi formada por camadas de elementos a partir da extrusão da malha inicial plana, sendo que a medida que se afasta do centro do mesmo as camadas aumentam de tamanho chegando a seu maior valor na superfície. Entre 15 e 22 camadas foram utilizadas na espessura, já que demonstraram convergência ao objetivo pretendido em trabalhos de Nevalainen e Dodds [16] e Cravero [17]. Vale ressaltar que os modelos 3D em EPD apresentaram em torno de 3200 elementos e 6800 nós para os corpos de prova $\mathrm{SE}(\mathrm{T}), 1300$ elementos e 2800 nós para os corpos de prova $\mathrm{SE}(\mathrm{B})$ e 4200 elementos e 8800 nós para os corpos de prova $\mathrm{C}(\mathrm{T})$, enquanto que, análises dos modelos 3D pleno $(W=B, W=2 B$ e $W=4 B$ ) apresentaram até 92000 elementos e 100000 nós. O carregamento foi realizado por deslocamentos incrementais para favorecer a convergência numérica da solução iterativa.

\subsubsection{Modelos Modified Boundary Layer (MBL)}

A descrição dos campos de tensões e deformações pela formulação HRR apresenta inconsistência física quando o raio de arredondamento da trinca tende a zero, pois a tensão gerada pelo carregamento tende ao infinito. Além disso, as formulações HRR (baseadas em teoria de pequenas deformações) não levam em consideração o arredondamento de ponta da trinca quando o mesmo apresenta plasticidade relevante [1]. Em face de tais argumentos e visando facilitar a geração dos campos de tensões de alta restrição plástica e alta triaxialidade (análogos à solução HRR) que possam ser usados como referência ao trabalho, foram desenvolvidos modelos Modified Boundary Layer (MBL) ou modelo da camada limite [16]. Tais modelos foram elaborados com base em um campo de deslocamentos remoto prescrito para ser representativo de uma estrutura ou amostra com dimensões características que garantem elevada restrição à plasticidade. É possível garantir tais fatores (alta restrição plástica) sem comprometer a descrição da plasticidade local, considerando uma placa semi-infinita, deslocamentos na espessura igual a zero $(w=0)$ e trações remotas dadas pelos primeiros dois termos da solução linear de Williams [18]. 


\subsection{Matriz de análise desenvolvida}

A tabela 2 apresenta a matriz de análise desenvolvida respectivamente para corpos de prova $\mathrm{C}(\mathrm{T}), \mathrm{SE}(\mathrm{B})$ e $\mathrm{SE}(\mathrm{T}) \mathrm{com} \mathrm{H} / \mathrm{W}=10$ solicitado por garras. Fica evidente a grande abrangência das análises em termos de profundidades relativas de trincas, propriedades de materiais e espessuras $B$.

Tabela 2. Matriz de análise desenvolvida para os corpos de prova.

\begin{tabular}{ccccc}
\hline $\boldsymbol{a} / \boldsymbol{W}$ & $\boldsymbol{n}$ & $\begin{array}{c}\text { Espessuras } \\
(\mathbf{m m})\end{array}$ & Corpos de prova & $\begin{array}{c}\text { Quantidade } \\
\text { de modelos }\end{array}$ \\
\hline 0,$1 ; 0,2 ; 0,3 ; 0,4 ; 0,5 ; 0,6 ; 0,7$ & $5 ; 10 ; 20$ & $\begin{array}{c}\mathrm{EPD} ; 12,5 ; \\
25,4 ; 50,8\end{array}$ & $\mathrm{C}(\mathrm{T}), \mathrm{SE}(\mathrm{B})$, & $\mathrm{SE}(\mathrm{T})-\mathrm{H} / \mathrm{W}=10$ \\
252 modelos \\
\hline
\end{tabular}

\subsection{Determinação numérica dos limites de deformação $(M)$}

Por meio de análises elásto-plásticas descritas anteriormente, torna-se possível avaliar os limites de deformação $M$ para diferentes geometrias de corpos de prova. Para cada nível de $J$ imposto, a determinação do limite de deformação $M$ decorre da comparação entre as tensões que ocorrem ao longo do ligamento remanescente (representado pela dimensão $b$ na figura 2) dos modelos elasto-plásticos dos corpos de provas com as tensões presentes no ligamento remanescente do modelo MBL (figura 5). Quando as tensões nos corpos de prova se desviam de um determinado percentual do campo de referência do $\mathrm{MBL}$, o valor de $M$ é aferido utilizando a equação 1. A fim de facilitar tal comparação foram empregadas evoluções da tensão normalizada $v s$. raio normalizado a partir da ponta da trinca $[1,17]$, sendo a normalização da tensão dada pelo limite de escoamento do material e a normalização do raio a partir da trinca dada pela razão entre o limite de escoamento e o valor de integral $J$ em domínio onde seu valor já seja independente do domínio de integração.

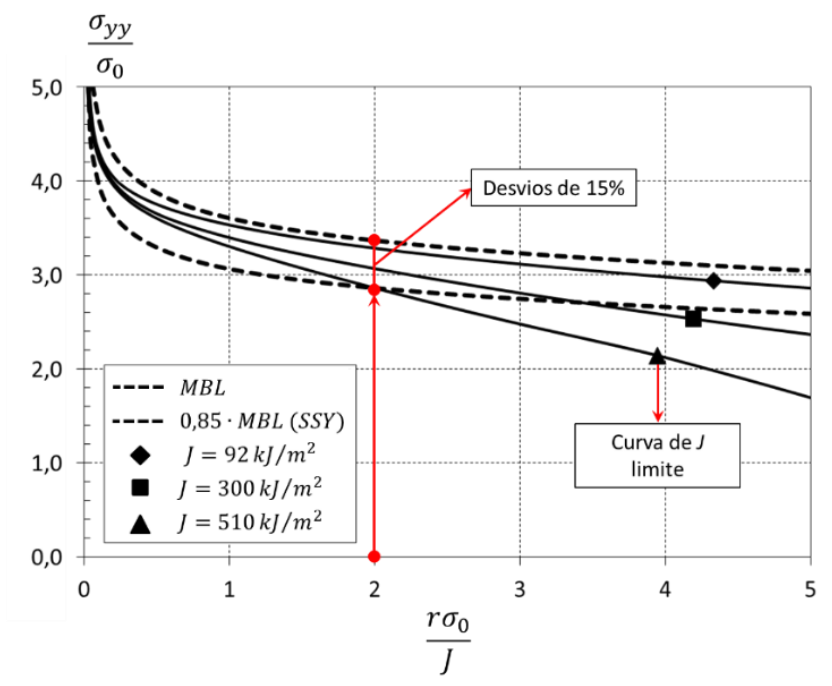

Figura 5. Exemplo de resultado obtido para análise do parâmetro $M$, considerando o procedimento anterior, salvo que para o caso está sendo considerado um raio adimensional igual a 2 e $n=10$.

Considerando um resultado típico para fins de exemplificação, verifica-se pela figura 5 que utilizando valores de $J$ iguais a $92 \mathrm{~kJ} / \mathrm{m}^{2}$ (considerado baixo), existe boa aderência entre as curvas dos campos de tensões no corpo de provas com o MBL, enquanto que utilizando valores de 300 e $510 \mathrm{~kJ} / \mathrm{m}^{2}$ (considerados altos) existe perda considerável de aderência entre as curvas. A partir do momento em que as tensões 
do modelo começam a apresentar desvios expressivos (no caso ilustrado de $15 \%$ ) em relação às tensões do modelo $\mathrm{MBL}$, para um dado valor de raio adimensional $r$, o valor da integral $J$ pode ser considerado naquele momento como limítrofe para a validade da MFEP monoparamétrica.

Vale ressaltar que toda esta metodologia foi incorporada em uma rotina desenvolvida pelo autor utilizando linguagem Matlab ${ }^{\circledR}$ (2013) e os resultados obtidos são totalmente congruentes com alguns resultados pontuais encontrados na literatura.

\section{RESULTADOS E DISCUSSÕES}
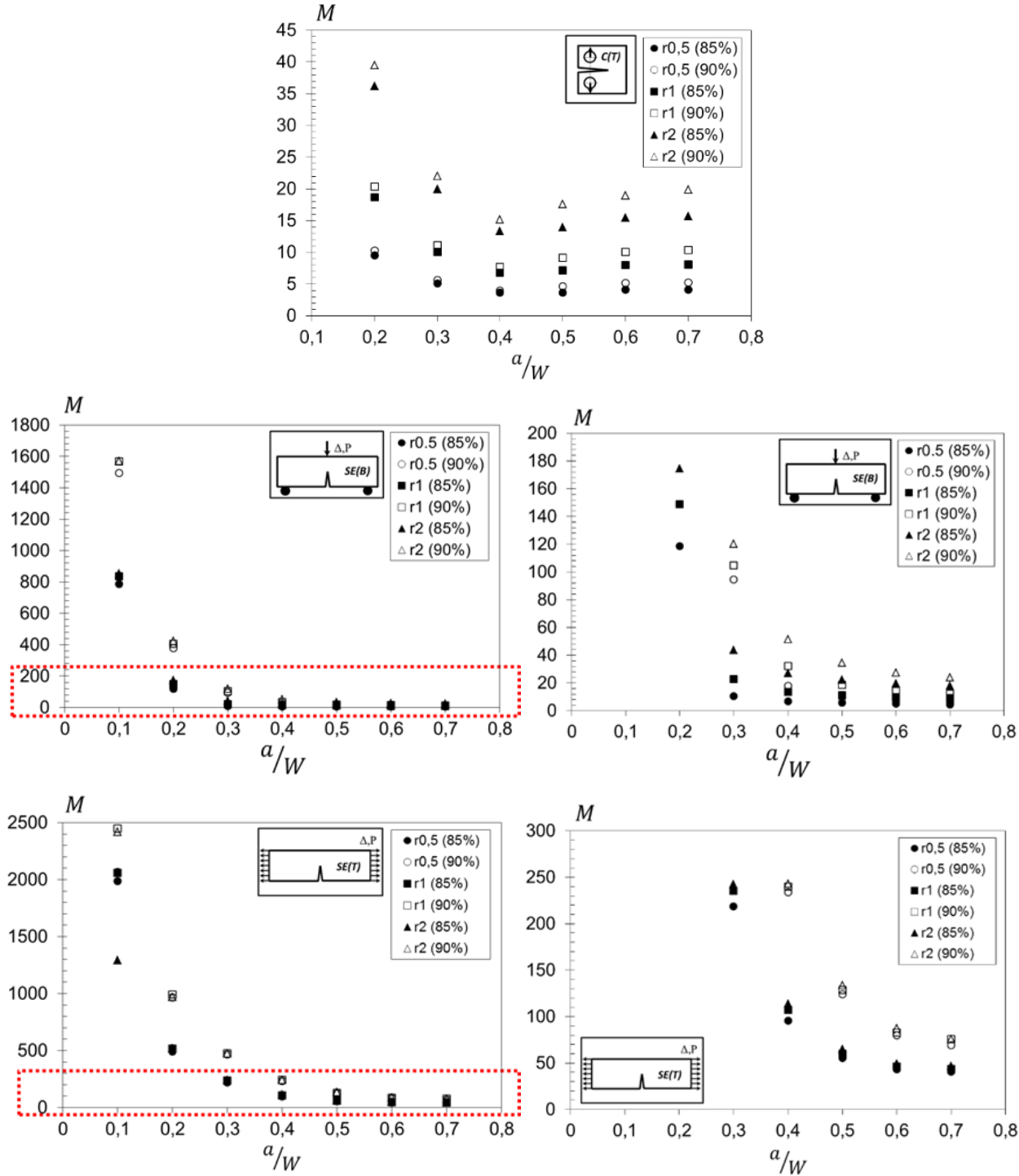

Figura 6. Influência de $a / W$ no comportamento do parâmetro $M$ em corpos de prova $\mathrm{C}(\mathrm{T}), \mathrm{SE}(\mathrm{B}) \mathrm{e}$ $\mathrm{SE}(\mathrm{T})-H / W=10$, considerando EPD e $n=10$. A região destacada em vermelho foi ampliada nas figuras da direita para melhor visualização.

Conforme pode ser observado na figura 6 para $n=10$ e EPD, em todos os tipos de geometrias de corpo de prova existe forte depêndencia não linear do parâmetro $M$ em relação ao comprimento relativo de trinca $(a / W)$. Ainda, fica evidente que $M$ é sensível ao raio adimensional no qual é avaliado (nas imagens são apresentados resultados para $r=0,5, r=1$ e $r=2$ ) e ao critério de desvio percentual adotado em relação ao campo de referência dos modelos MBL (10\% e 15\%). Além disso, verificou-se que, 
para todos os corpos de prova em estudo, o aumento do comprimento relativo da trinca causa um decréscimo expressivo do parâmetro $M$ (gerando maiores $J_{\text {lim }}$, ou seja, ampliando a validade da MFEP em trincas profundas - condizente com as normas correntes), principalmente para $0,4 \geq a / W \geq 0,7$. Exceção deve ser feita para a geometria $\mathrm{C}(\mathrm{T})$, já que para tais profundidades ocorre leve incremento no valor do $M$, o que foi explicado por investigações paralelas das trajetórias $J-Q$ obtidas para diferentes $a / W$ - para $a / W=0,7$ [19] existe uma inversão de tendência da trajetória $J-Q$ e leve perda de restrição à plasticidade em relação a $a / W=0,5$.

Com relação aos corpos de prova $\mathrm{C}(\mathrm{T})$ e $\mathrm{SE}(\mathrm{B})$ (figura 6) e considerando a mesma relação $a / W$, as tensões normalizadas para diferentes raios adimensionais de avaliação (no caso 0,5, 1 e 2) apresentam comportamentos distintos para o parâmetro $M$, enquanto que corpos de prova $S E(T)$, exceto para trincas rasas $(a / W<0,3)$, apresentam grande aderência entre os valores.

Avaliando-se agora o efeito de $n=5,10$ e 20 (figura 7), constatou-se para corpos de prova $\mathrm{C}(\mathrm{T})$ que independentemente de $a / W$, os desvios percentuais entre os valores de $M$ para diferentes encruamentos são expressivos. Já com relação aos corpos de prova $\mathrm{SE}(\mathrm{B})$ e $\mathrm{SE}(\mathrm{T})$, observou-se também expressiva variação dos valores de $M$ para diferentes relações de $a / W$ e níveis de encruamento, embora em alguns casos a visualização seja dificultada dados os altos valores de $M$ para trincas rasas.


Figura 7. Influência de a/W no comportamento do parâmetro $M$ em corpos de prova $C(T), S E(B) e$ $\mathrm{SE}(\mathrm{T})-\mathrm{H} / \mathrm{W}=10$, considerando $n=5,10$ e 20 e $10 \%$ de desvio. 
Por questões de espaço, para mostrar as tendências dos valores de $M$ incluindo o efeito das espessuras consideradas na matriz de análise desse trabalho, optou-se por trabalhar com os dados de raio adimensional igual a 2 e desvios de $10 \%$ em relação à referência (modelo MBL). Os resultados centrais encontram-se na figura 8 .

Fica evidente na figura 8 que corpos de prova $\mathrm{C}(\mathrm{T})$ seguem a tendência esperada para comprimentos relativos de trinca entre $0,2 \leq a / W \leq 0,5$. Fica também evidente uma grande diferença entre os valores de $M$ para diferentes configurações de $a / W$ e $W / B$ e que a não linearidade dos valores de $M$ em relação a $a / W$ é mantida assim como nos casos em EPD, exceto pela condição de $W=4 B$ em que a tendência de evolução dos valores de $M$ é praticamente linear. Um fenômeno curioso ocorre para trincas com $a / W$ $>0,5$ pois os valores de $M$ de um corpo de prova $\mathrm{C}(\mathrm{T})$ mais espesso $(W=B)$ se revelam maiores que de um menos espesso $(W=2 B)$. O significado físico disto é que neste caso uma amostra com trinca profunda teria maior restrição plástica com espessura de $25,4 \mathrm{~mm}$ do que de $50,8 \mathrm{~mm}$. Por meio de trajetórias $J$ - $Q$ avaliadas em paralelo ao trabalho [19], verificou-se que tal fenômeno de fato foi observado e provavelmente se deve às proporções de $b / W$ propiciadas somente com a alteração de espessura.

No caso de corpos de prova $S E(B)$, duas questões interessantes podem ser avaliadas a partir da figura 8. A primeira é que para trincas rasas $(a / W \leq 0,2)$ corpos de prova mais finos $(W=4 B)$ apresentam maior restrição à plasticidade em relação a mais espessos $(W=B)$. A segunda questão é semelhante à apresentada anteriormente para corpos de prova $\mathrm{C}(\mathrm{T})$ em que para trincas muito profundas $(a / W \geq 0,6)$ a restrição plástica em corpos de prova mais finos é maior que mais espessos. A primeira questão pode ter a ver com a distância da ponta da trinca em relação à linha neutra do corpo de prova, pois trincas rasas apresentam carregamento local predominantemente trativo e isto faz com que a restrição à plasticidade possa diminuir. A segunda questão pode ser explicada da mesma forma que a de corpos de prova $\mathrm{C}(\mathrm{T})$ por meio de trajetórias $J-Q$ [19].

Por fim, corpos de prova $\mathrm{SE}(\mathrm{T})$ apresentam tendências semelhantes às dos corpos de prova $S E(B)$, mas a inversão de tendência para trincas rasas se mostra ainda mais marcante $(a / W<0,3)$ devido ao fato de esta geometria ter carregamento intrinsecamente trativo para todos as relações de $a / W$. 

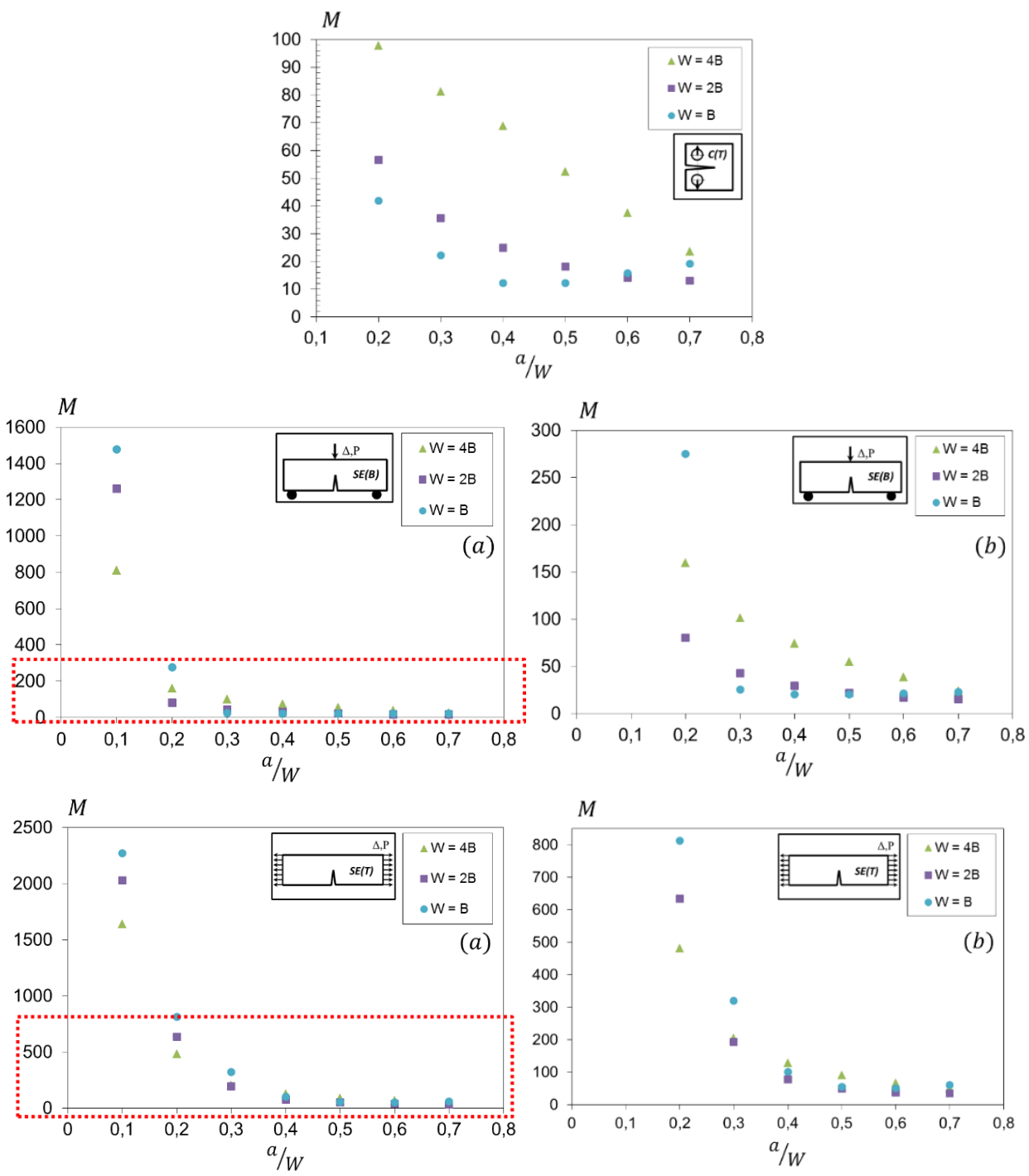

Figura 8. Influência do comprimento relativo de trinca no comportamento do parâmetro $M$ em corpos de prova $\mathrm{C}(\mathrm{T}), \mathrm{SE}(\mathrm{B})$ e $\mathrm{SE}(\mathrm{T})-\mathrm{H} / \mathrm{W}=10$, considerando $n=10$ e $\mathrm{W} / B=1,2$ e 4 .

\section{CONCLUSÕES}

A partir dos resultados obtidos, as seguintes conclusões podem ser apresentadas:

- Os valores do limite de deformação $M$ são muito sensíveis a diferentes tipos de geometrias, modos de carregamento e profundidades relativas de trincas. Isto impacta diretamente na adequação e extensão de validade de propriedades de tenacidade à fratura obtidas em laboratório transferíveis a estruturas reais com segurança e realismo.

- Os resultados apresentados servem de base para a verificação de validade da Mecânica da Fratura Elasto Plástica em corpos de provas de uso corrente. E mesmo no caso de invalidação da dominância $J$, os resultados de $M$ (e consequentemente $J_{\text {lim }}$ ) apresentados servem de base para a avaliação quantitativa da necessidade de emprego de metodologias bi-paramétricas e propriedades mecânicas dependentes de geometria.

- Os valores obtidos para o parâmetro $M$ estão em acordo com os fundamentos da teoria de mecânica da fratura e argumentos da teoria $J$ - $Q$, a qual demonstra que corpos de prova $\mathrm{C}(\mathrm{T})$ apresentam elevada restrição à plasticidade 
enquanto na direção oposta, corpos de prova $S E(T)$ apresentam baixa restrição à plasticidade devido a seu carregamento predominantemente trativo.

- Verificou-se por meio das análises de corpos de prova com efeito 3D a necessidade de cautela quanto a generalizações relacionadas ao ganho ou perda de restrição plástica quando características geométricas são alteradas. Ficou evidente que casos de trincas muito rasas e muito profundas podem inverter as tendências esperadas de ganho de restrição plástica com aumento da espessura.

- No geral, os extensivos resultados de $M$ apresentados, se tomados complementarmente às recomendações existentes em normas correntes, podem aprimorar sensivelmente a validação das propriedades de tenacidade à fratura experimentais e as decisões sobre a necessidade de emprego de metodologias bi-paramétricas ou propriedades dependentes da geometria.

\section{Agradecimentos}

Este trabalho é suportado pelo Centro Universitário da FEI por meio de recursos materiais, humanos e infra-estrutura laboratorial. Adicionalmente, os autores gostariam de agradecer e à CAPES pela bolsa de mestrado concedida ao aluno FCM.

\section{REFERÊNCIAS}

[1] ANDERSON, T. L. Fracture Mechanics: fundamentals and applications. 3. ed. New York: CRC, 2005.

[2] RICE, J. R. A path independent integral and the approximate analysis of strain concentration by notches and cracks. Journal of Applied Mechanics, v. 35, p. 379-386, 1968.

[3] LANDES, J. D. ; BEGLEY, J. A. The J integral as a fracture criterion. ASTM STP 514. Philadelphia, p. 1-20, 1972.

[4] RICE, J. R; ROSENGREN, G. F. Plane strain deformation near a crack tip in a powerlaw hardening material. Journal of the Mechanics and Physics of Solids, v. 16, p. 1-12, 1968.

[5] HUTCHINSON, J. W. Singular behavior at the end of a tensile crack tip in a hardening material. Journal of the Mechanics and Physics of Solids, v. 16, p. 13-31, 1968.

[6] RICE, J. R ; ROSENGREN, G. F. Plane strain deformation near a crack tip in a powerlaw hardening material. Journal of the Mechanics and Physics of Solids, v. 16, p. 1-12, 1968.

[7] PARIS, P. C., Discussion in fracture toughness. ASTM STP 514. Philadelphia, 1972. p. 21-22.

[8] LANDES, J. D. ; BEGLEY, J. A. Tests results from J-Integral studies: an attempt to establish a $J_{I C}$ testing procedure. ASTM STP 560. Philadelphia, p. 170-186, 1974.

[9] LANDES, J. D. ; BEGLEY, J. A. Serenditipy and the J-Integral. International Journal of Fracture Mechanics, v. 12, p. 764-766, 1976.

[10] MCMEEKING, R. M. Finite deformation analysis of crack tip opening in elastic-plastic materials and implications for fracture. Journal of Mechanics and Physics of Solids, v. 25, p. 357-381, 1977.

[11] SHIH, C.F. ; GERMAN, M.D. Requirements for a one parameter characterization of crack tip fields by the HRR singularity. International Journal of Fracture, v. 17, p. 27-43, 1981.

[12] AMERICAN SOCIETY FOR TESTING AND MATERIALS. ASTM E1820: standard test method for measurement of fracture toughness. Philadelphia, junho 2011.

[13] DET NORSKE VERITAS. DNV-RP-F108: fracture control for pipeline installation methods introducing plastic strain. Norway, 2012. 
[14] KOPPENHOEFER, K. et al. WARP3D: Dynamic nonlinear analysis of solids using a preconditioned conjugate gradient software architecture. Structural Research Series (SRS) 596. University of Illinois at Urbana-Champaign, 1994.

[15] MSC SOFTWARE CORPORATION. Patran. Disponível em: <http://www.mscsoftware. com /products/patran>. Acesso em: 10 abr 2012.

[16] NEVALAINEN, M. ; DODDS JR, R. H. Numerical investigation of 3-D constraint effects on brittle fracture in $\mathrm{SE}(\mathrm{B})$ and $\mathrm{C}(\mathrm{T})$ specimens. International Journal of Fracture, v. 74, p. 131-161, 1995.

[17] CRAVERO, S. Desenvolvimento de procedimentos para avaliação de curvas J-R em espécimes à fratuta $S E(T)$ utilizando o método de flexibilidade. 2007. $162 \mathrm{f}$. Tese (Doutorado em Engenharia) - Escola Politécnica, USP, São Paulo.

[18] WILLIAMS, M. L. On the stress distribution at the base of a stationary crack. Journal of Applied Mechanics, v. 24, p. 109-114, 1957.

[19] MOREIRA, F. C. Determinação numérica de limites de deformação e flexibilidades elásticas aplicáveis a geometrias C(T), SE(B) e SE(T). 2014. Dissertação (Mestrado em Engenharia) - Centro Universitário da FEI, São Bernardo do Campo, SP 